\title{
La dynamique des particules de caséine de diamètres différents dans le lait de brebis au cours de la lactation
}

\author{
par \\ Dr Jovan DJORDJEVIC, \\ Faculté d'Agriculture de Belgrade - Zemun \\ et M. Marijana CARIC, \\ Faculté de Technologie de Novi Sad
}

Les résultats obtenus à l'aide de la microscopie électronique dans l'étude des particules de la caséine représentaient une stimulation de plus pour continuer les recherches intensives de cette protéine du lait. En utilisant cette méthode, Nitschmann [12] a constaté une fois de plus la polydispersion de la caséine. Hostettler et Imhoff [9] ont réussi à établir l'influence des différents facteurs, en premier lieu celle du calcium, sur le diamètre et la forme des globules de la caséine. Grâce à ces découvertes, ils ont expliqué plusieurs phénomènes concernant les propriétés technologiques du lait. Cette découverte était suivie de plusieurs travaux qui traitaient de l'influence de l'espèce [11], de la nutrition [1] et de la saison [2] sur le diamètre des particules de la caséine.

La grande majorité des travaux dans le domaine se rapporte au lait de vache tandis que les données concernant le lait de brebis sont très rares $[4,6,7]$. Ce fait nous a incité à rechercher la répartition des particules de la caséine de diamètres différents dans le lait de brebis sous l'influence de la période de lactation, ce qui est le sujet du présent travail.

La caséine se trouve dans le lait sous forme d'une association complexe qui renferme dans sa composition certains constituants minéraux : en premier lieu, le calcium, le magnésium et les phosphates. Plusieurs auteurs [3] ont constaté que la quantité de ces composants dans la micelle de caséine n'est pas constante, ce qu'on peut voir d'après les variations des rapports calcium/azote ou phosphore/ azote de la caséine. Les résultats de Hostettler et al. [10] montrent que la quantité de calcium s'accroît de 2,61 à 3,66 p. 100 quand le diamètre des particules de la caséine augmente. Pour cette raison, nous avons suivi la quantité des diverses formes du calcium dans le 
lait de brebis au cours de la lactation pour voir s'il existait une corrélation avec les dimensions des particules de la caséine.

\section{PROCEDE EXPERIMENTAL}

Prise d'échantillons. Les brebis de la race tsigaya, qui furent dans les mêmes conditions d'élevage au cours de toute la période de recherches, étaient réparties par groupes de cinq, et les échantillons étaient composés du lait d'un groupe. On prenait les échantillons tous les $14 \mathrm{j}$ pendant la lactation.

Ecrémage. Avant l'ultracentrifugation on éliminait à l'aide d'une écrémeuse 99 p. 100 de la matière grasse des échantillons.

Séparation des particules de la caséine de diamètres différents au cours de la lactation. Pour obtenir la séparation des micelles de caséine de diamètres différents on a soumis le lait écrémé à l'ultracentrifugation différentielle. On s'est servi de trois forces centrifuges : de $10300 \mathrm{xg}$, de $47600 \mathrm{x}$ g et de $144400 \mathrm{x}$ g ce qui correspond à 10000 , 21500 et $38000 \mathrm{t} / \mathrm{mn}$. La quantité d'azote dans le sédiment a été déterminée par la méthode microkjeldahl.

L'ultracentrifuge qui a été employée dans ces expériences est le modèle VAC 60 de la maison « Janetzki ». On a employé le rotor angulaire dont le volume déclaré est 6 fois $50 \mathrm{ml}$ et le nombre maximum de $\mathrm{t} / \mathrm{mn}$ est de 40000 , ce qui correspond à la force de $160000 \mathrm{xg}$. L'ultracentrifugation se faisait pendant $1 \mathrm{~h}$ à la température variant de $0^{\circ} \mathrm{C}$ à $5^{\circ} \mathrm{C}$.

Dans l'ultracentrifugat qui, pratiquement, ne contenait plus de caséine, on a déterminé le calcium qui, en fait, représente la quantité de cet élément existant dans le lait sous forme soluble, c'est-à-dire, qui n'appartient pas au phosphocaséinate de chaux.

\section{RESULTATS ET DISCUSSION}

Les forces centrifuges de $10300 \mathrm{~g}, 47600 \mathrm{~g}$ et $144400 \mathrm{~g}$, qu'on a employées au cours des expériences, ont été choisies d'après la courbe de distribution par grandeur des globules de la caséine [4]. De la

$$
\begin{aligned}
\text { relation } \mathrm{d} & =\sqrt{\frac{60}{4 n^{2}} \cdot \frac{\ln \mathrm{R}_{\max .}-\ln \mathrm{R}_{\min .}}{n^{2}} \cdot \frac{18 \mathrm{n} \bar{v}}{(1-\bar{v} \sigma) \mathrm{T}_{\mathrm{s}}}} \\
\mathrm{R}_{\max .} & =\text { le diamètre maximum du rotor }(\mathrm{cm}) \\
\mathrm{R}_{\min .} & =\text { le diamètre minimum du rotor }(\mathrm{cm}) \\
\mathrm{n} & =\text { le nombre de rotations par minute }(\mathrm{r} / \mathrm{mn}) \\
\sigma & =\text { densité du milieu }\left(\mathrm{g} / \mathrm{cm}^{3}\right) \\
\frac{\mathrm{n}}{v} & =\text { viscosité du milieu }(\mathrm{cP}) \\
\mathrm{T}_{s} & =\text { volume spécifique partiel } \\
& =\text { temps de sédimentation }(\mathrm{sec})
\end{aligned}
$$


on trouve qu'en utilisant la force de $10300 \mathrm{~g}$ on sépare les globules dont le diamètre dépasse $140,7 \mathrm{~m} \mu$; la force de $47600 \mathrm{~g}$ retient les micelles dont le diamètre se range entre 65,5 et $140,7 \mathrm{~m} \mu$; tandis que les globules dont le diamètre se trouve entre 37,2 et $65,5 \mathrm{~m} \mu$ sont séparées par une force atteignant $144400 \mathrm{~g}$. Les globules inférieurs à 37,2 $\mathrm{m} \mu$ restent dans l'ultracentrifugation, mais leur quantité est minime car il s'agit de 0,02 à 0,03 p. 100 d'azote de la caséine.

\section{fig. 1}

Proportion des globules de caséine de diamètres différents au cours de la lactation

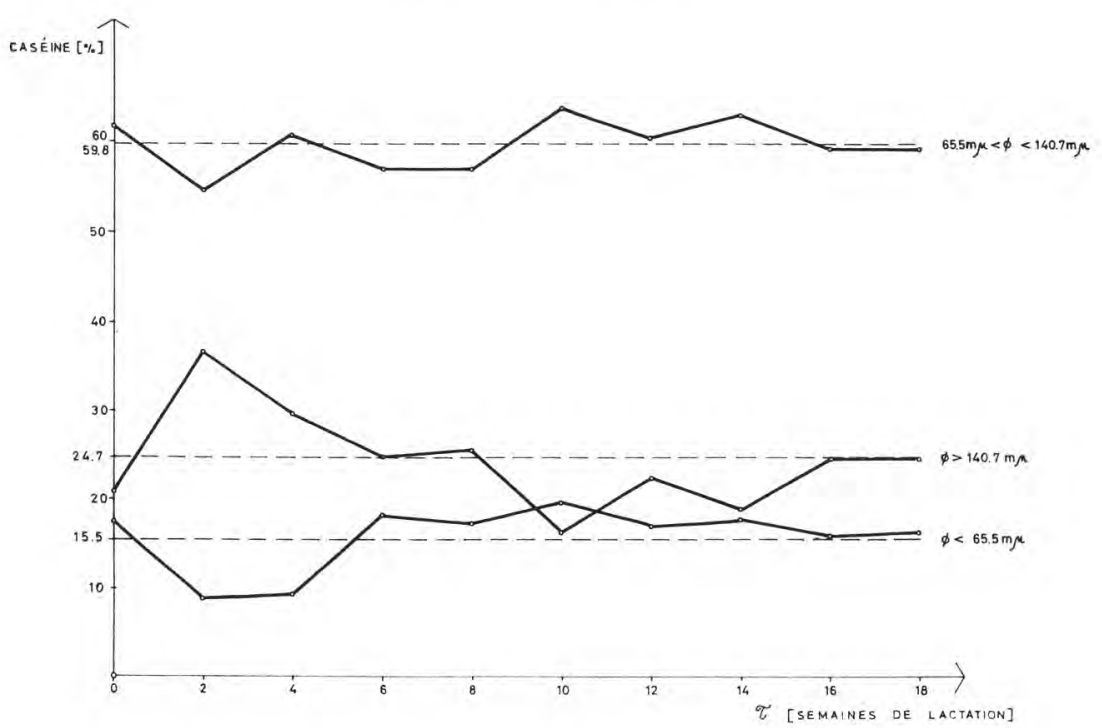

Les résultats présentés dans le tableau 1 et la figure 1 montrent qu'environ 60 p. 100 de la caséine totale est constitué par des particules dont le diamètre se trouve entre 65,5 et $140,7 \mathrm{~m} \mu$. Les plus grandes particules font 24,7 et les plus petites 15,5 p. 100.

Si l'on suit la dynamique de différentes catégories au cours de la lactation on peut constater :

a) Les plus grandes micelles, dont le diamètre dépasse 140,7 $\mathrm{m} \mu$, sont les plus nombreuses au commencement de la lactation, quand elles atteignent même 36,6 p. 100 de la caséine totale. Avec l'avancement de la lactation leur participation diminue et à la fin de la $10^{\mathrm{me}}$ semaine elles atteignent leur minimum et ne font que 16,3 p. 100 de la caséine totale. Vers la fin de la lactation la quantité de cette catégorie de particule augmente, quoique irrégulièrement, et atteint 24,5 p. 100 de la caséine du lait de brebis. 
b) La proportion des plus petites particules dans la caséine totale montre une tendance presque inverse en comparaison avec les plus grandes. Leur proportion, qui est en absolu la plus petite, augmente du commencement au milieu de la lactation. La proportion de cette catégorie au milieu de la lactation dépasse de 55 p. 100 celle au cours du premier tiers.

c) La catégorie des particules la plus représentée (65,5 à 140,7 $\mathrm{m \mu})$ ne montre pas une régularité de changement au cours de la lactation, quoiqu'on puisse constater une proportion minimum vers le commencement et une valeur maximum vers le milieu de la lactation. La figure 1 révèle que la proportion de cette catégorie de particules est plus petite dans la première que dans la seconde moitié de la lactation.

TABLEAU 1. - Proportion des globules de la caséine à diamètres différents au cours de la lactation

\begin{tabular}{|c|c|c|c|}
\hline \multirow{2}{*}{$\begin{array}{l}\text { Analyse au cours } \\
\text { de la } \\
\text { lactation }\left[\mathrm{N}^{\circ}\right]\end{array}$} & \multicolumn{3}{|c|}{ Caséine [p. 100] } \\
\hline & $\varnothing>140.7 \mathrm{~m} \mu$ & $65.5<\varnothing<140.7 \mathrm{~m} \mu$ & $\varnothing<65.5 \mathrm{~m} \mu$ \\
\hline 1 & 20.7 & 62.0 & 17.3 \\
\hline 2 & 36.6 & 54.7 & 8.7 \\
\hline 3 & 29.7 & 61.0 & 9.3 \\
\hline 4 & 24.7 & 57.2 & 18.1 \\
\hline 5 & 25.6 & 57.2 & 17.2 \\
\hline 6 & 16.3 & 64.1 & 19.6 \\
\hline 7 & 22.2 & 60.7 & 17.1 \\
\hline 8 & 18.8 & 63.4 & 17.8 \\
\hline 9 & 24.5 & 59.6 & 15.9 \\
\hline 10 & 24.5 & 59.3 & 16.2 \\
\hline $\bar{x}$ & 24.7 & 59,8 & 15.5 \\
\hline
\end{tabular}

Pour résumer, on peut dire que les oscillations sont plus prononcées au début de la lactation, tandis que plus tard les valeurs deviennent plus stables et elles varient dans des limites bien plus étroites. Les grandes variations au début de la lactation favorisent la proportion des globules dont le diamètre dépasse 140,7 mu par rapport aux 
deux autres catégories de micelles. Tout cela nous amène à conclure que les 6 premières semaines de lactation représentent la période d'une stabilisation relative non seulement de la composition globale du lait, comme on le sait, mais aussi de la répartition de globules de la caséine.

On sait que le calcium fait partie de la composition et des liaisons de la particule de la caséine. Hostettler et al. [10] nous enseignent que les particules de diamètre supérieur contiennent plus de calcium. C'est pourquoi on voulait voir s'il y avait rapport entre la quantité du calcium colloïdal et soluble et la proportion relative des micelles de la caséine, d'autant plus que dans la littérature on ne rencontre pas

fig. 2

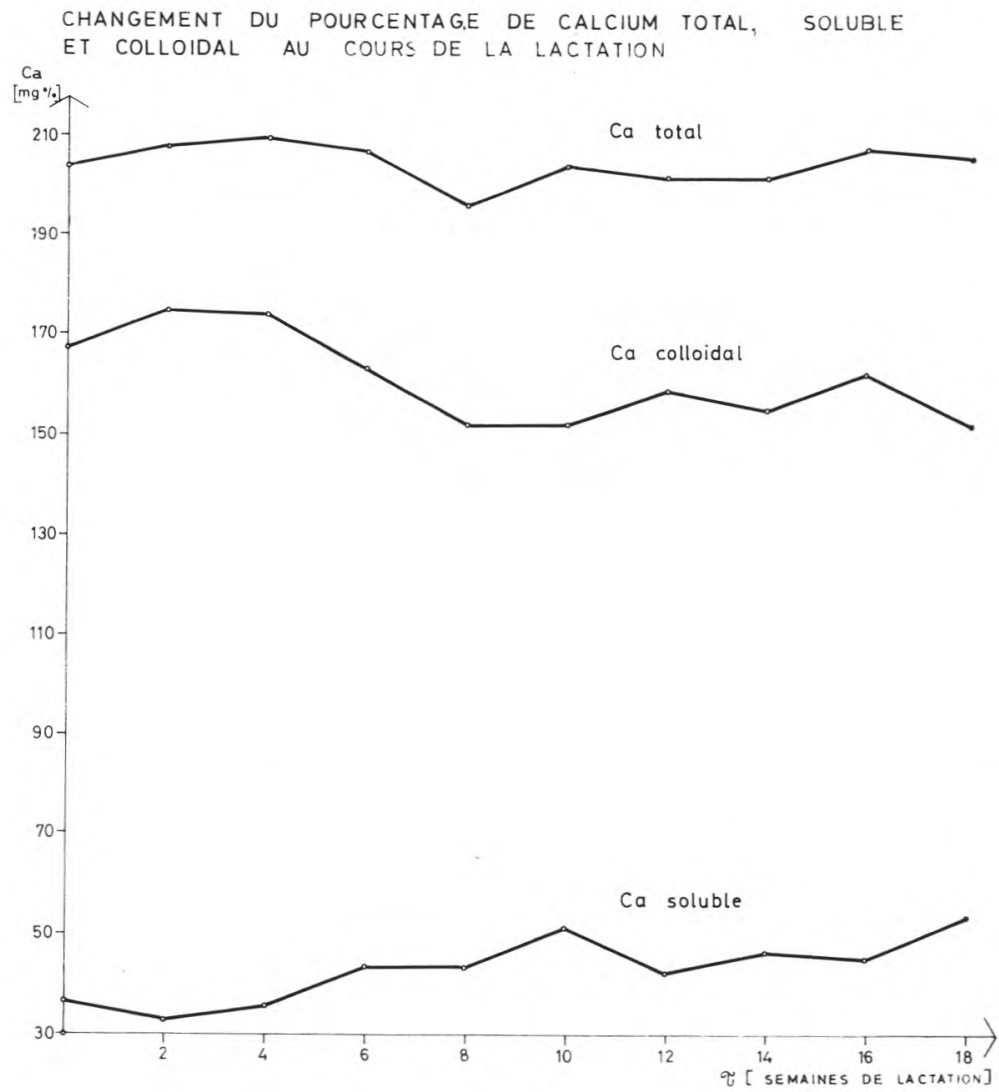


TABLEAU 2. - Changement du pourcentage de calcium total, soluble et colloïdal au cours de la lactation

\begin{tabular}{|c|c|c|c|c|c|c|}
\hline $\begin{array}{c}\text { Analyse } \\
\text { au cours de la } \\
\text { lactation } \\
{\left[\mathrm{N}^{\circ}\right]}\end{array}$ & $\begin{array}{c}\text { Ca total } \\
{[\mathrm{mg} \mathrm{p.} \mathrm{100]}}\end{array}$ & $\begin{array}{l}\text { Ca soluble } \\
\text { [mg p. 100] }\end{array}$ & $\begin{array}{l}\text { Ca colloïdal } \\
\text { [mg p. 100] }\end{array}$ & $\begin{array}{c}\text { Proportion de la } \\
\text { forme soluble } \\
\text { dans le Ca total } \\
\text { [p. 100] }\end{array}$ & $\begin{array}{c}\text { Proportion de la } \\
\text { forme colloïdale } \\
\text { dans le Ca total } \\
\text { [p. 100] }\end{array}$ & $\begin{array}{l}\text { Calcium } \\
\text { de la } \\
\text { caséine } \\
\text { [p. 100] }\end{array}$ \\
\hline 1 & 204 & 36.6 & 167.4 & 17.9 & 82.1 & 2.70 \\
\hline 2 & 208 & 33.0 & 175.0 & 15.9 & 84.1 & 2.78 \\
\hline 3 & 210 & 35.9 & 174.1 & 17.1 & 82.9 & 2.44 \\
\hline 4 & 207 & 43.8 & 163.2 & 21.2 & 78.8 & 2.45 \\
\hline 5 & 196 & 43.4 & 152.6 & 22.2 & 77.8 & 2.66 \\
\hline 6 & 204 & 51.7 & 152.3 & 25.2 & 74.8 & 2.45 \\
\hline 7 & 202 & 42.7 & 159.3 & 21.2 & 78.8 & 2.54 \\
\hline 8 & 202 & 46.8 & 155.2 & 23.2 & 76.8 & 2.29 \\
\hline 9 & 208 & 45.4 & 162.6 & 21.8 & 78.2 & 2.25 \\
\hline 10 & 206 & 53.9 & 152.1 & 26.2 & 73.8 & 2.30 \\
\hline $\bar{x}$ & 204 & 43.3 & 161.4 & 21.2 & 78.8 & 2.49 \\
\hline
\end{tabular}


de résultats qui indiqueraient le pourcentage de calcium de lait de brebis lié à la caséine.

Le liquide obtenu à la fin de l'ultracentrifugation contient de 33,0 à $53,9 \mathrm{mg}$ p. 100 de calcium avec une moyenne de $43,3 \mathrm{mg}$ p. 100 pour toute la période des expériences. Ce calcium soluble représente 21,2 p. 100 du calcium total, ce qui est relativement moindre que dans le lait de vache [5].

Le calcium colloïdal, séparé avec la caséine, varie de 152,1 à $175,0 \mathrm{mg}$ p. 100 avec une moyenne de 161,4 . Les quantités des différentes formes du calcium au cours de la lactation sont présentées dans le tableau 2 et la figure 2.

Les courbes qui représentent la dynamique du calcium total et colloïdal ont des marches analogues, ce qui confirme une fois de plus que la quantité du calcium colloïdal dépend aussi de la teneur totale en cet élément.

La marche de la courbe révèle que le lait de brebis contient le plus de calcium colloïdal au début et le moins au milieu de la lactation, ce qui coïncide avec la proportion maximum et minimum des plus grandes particules de la caséine. Vers la fin de la lactation la proportion de cette catégorie de particules augmente de nouveau, de même que la teneur en calcium colloïdal. Cependant, quand on considère les résultats concernant le pourcentage de calcium lié à la caséine on obtient une impression un peu différente. Ces différences sont la conséquence de la variation des quantités de caséine au cours de la lactation.

Le pourcentage de calcium lié à la caséine varie de 2,29 à 2,78 avec une moyenne de 2,49 p. 100 . Ces valeurs sont inférieures à celles que nous présentent Hostettler et al. [10] ainsi que Ford et Ramsdell [8]. Ceci semble expliquer partiellement le fait que le lait de brebis est moins sensible à la coagulation sous l'action de la chymosine que le lait de vache. La caséine du lait de brebis contient plus de calcium au commencement de la lactation, puis la quantité diminue quand la fin de la lactation se rapproche. Au cours du premier tiers de la lactation la caséine contient 2,64 p. 100 , au milieu 2,52 p. 100 et pendant le dernier tiers 2,28 p. 100 de calcium. Quand on compare ces résultats avec ceux de la proportion des plus grandes particules de la caséine on peut se rendre compte qu'au début et au milieu de la lactation il existe une corrélation entre ces deux facteurs. Ceci est en accord avec les données précitées. Mais cela n'est pas le cas quand on considère la fin de la lactation. Cela revient à dire que, au cours du dernier tiers de la lactation, la proportion des plus grandes particules augmente de nouveau, tandis que le contenu de la caséine en calcium continue à diminuer. Ces différences entre la dynamique du calcium colloïdal au cours de la lactation et le pourcentage de calcium lié à la caséine sont provoquées par une augmentation de quantité de la caséine vers la fin de la lactation des brebis. 


\section{CONCLUSION}

On a recherché l'influence de la période de lactation sur la proportion de trois catégories de particules de la caséine et sur la dynamique des différentes formes de calcium dans le lait de brebis.

On a constaté que les particules dont le diamètre se trouve entre 65,5 et $140,7 \mathrm{~m} \mu$ constituent environ 60 p. 100 de la quantité totale de la caséine. La proportion de cette catégorie ne montre pas une régularité de changement au cours de la lactation.

La proportion des plus grandes particules diminue vers le milieu de la lactation puis elle augmente irrégulièrement vers la fin.

Les variations, concernant la proportion des particules de la caséine sont les plus prononcées au début de la lactation et les 6 premières semaines représentent la période au cours de laquelle se constitue une stabilisation relative de cette proportion.

Il existe une corrélation entre la dynamique du calcium total et colloïdal. Le calcium colloïdal constitue 78,8 p. 100 du total.

La caséine du lait de brebis contient moins de calcium colloïdal $(2,49$ p. 100$)$ que la caséine du lait de vache.

Il existe une certaine analogie au cours de la lactation entre la dynamique des plus grandes particules de la caséine et la quantité de calcium colloïdal.

\section{S u $\mathbf{m} \mathbf{m}$ a r y}

The studies have been made in order to research the influence of the lactation period on the participation of the three various sized casein particles categories as well as on the dynamics of the different forms of calcium in sheep milk casein.

It was found out that the particles with the diameter between 65.5 and $140.7 \mathrm{~m} \mu$ make about $60 \mathrm{p} .100$ from the total casein quantity. The participation of that category does not show a regularity in changing during the lactation. The participation of the biggest particles decreases towards the middle of lactation, then it increases irregularly to the end of lactation.

The variations concerning the different sized casein particles participation are mostly expressed at the beginning of lactation, so that the first six weeks represent the period during which appears a certain stabilisation of this relation.

There is a correlation in the dynamics of the total and colloidal calcium content. The colloidal calcium makes 78.8 p. 100 of the total. 
The sheep milk casein contains less colloidal calcium (2.49 p. 100) than that of the cow.

There is a certain analogy in the dynamics of the biggest casein particles and the quantity of calcium in colloidal form during the lactation period.

Reçu pour publication en décembre 1971.

\section{Bibliographie}

[1] BeletKov (M. P.) et Lusnikov (A. N.) (1970). - Sostav i svoistva kazeina moloka pri vkljucenii v racion korov senaza iz ljucerni. Mol. prom., 31, 7, 21.

[2] Bernatonis (V.J) et Mickene (B. N.) (1968). - Izmenenie razmera castic kazeina v zavisimosti ot vremeni goda i porodi korov. Mol. prom., 29, 4, 17.

[3] Bohren (U. H.) and WenNeR (R. V.) (1966). - Natural state of milk proteins. I. Composition of the micellar and soluble casein of milk after centrifugal sedimentation. J. D. Sci., 7, 1213.

[4] Caric (M.) and DJoRdjevic (J.). - Size distribution of casein globules (sous presse).

[5] DJoRdJevic (J.) (1971). - Prelaz kalciuma u grus i njegova dinamika u toku proizvodnje kackavalja. Arh. Polj. Nauke, Beograd, 6, 241.

[6] DJoRdJevic (J.) and CARIC (M.) (1970). - The microelectrophoretic composition of sheep milk casein with different particle size. XVIII Intern. Dairy Congress, Sidney, A.2.1.

[7] DJoRdJevic (J.) et CARIC (M.) (1971). - La composition microélectrophorétique des micelles de la caséine du lait de brebis de diamètres différents. Le Lait, 509/510, 653.

[8] Ford (T. F.) and al. (1955). - Composition of the casein containing particles in milk. J. D. Sci., 38,843 .

[9] Hostettler (H.) und ImнoF (K.) (1951). - Elektronenoptische Untersuchungen über den Feinbau der Milch. Milchwiss., 6, 10, 351.

[10] Hostettler (H.) und al. (1949). - Untersuchungen über das in der Kuhmilch in nativen (genuinen) Zustand vorliegende Kasein. Landwirtsch. Jahrb. Schw., 31-67.

[11] Knopp (E.) und Wortmann (A.) (1960), - Zur Grossenverteilung der Caseinteilchen in Kuhlmich, Ziegenmilch und Frauenmilch, Milchwiss., 15, 6, 273.

[12] Nitschmann (H.) (1949). - Elektronenmikroskopisch Grossenbestimung der Calcium Caseinatteilchen in Kuhlmich, Helv. Chim. Acta., 22, 1258. 